\title{
Prospecção científica e tecnológica sobre o potencial de plantas com ação antibacteriana e antioxidante para o desenvolvimento de cosméticos
}

\author{
Scientific and technological prospection on the potential of plants with \\ antibacterial and antioxidant action for the development of cosmetics
}

Ana Paula Lacerda $\operatorname{Costa}^{1}$; Fernanda Braz de Jesus ${ }^{1}$; Roberta Maria Santos Ornellas ${ }^{1}$; Gabriele Marisco $^{1 *}$ (autor correspondente)

\begin{abstract}
${ }^{1}$ Universidade Estadual do Sudoeste da Bahia - UESB, Vitória da Conquista - Bahia, Brasil, 45083-900, 1acerdaana20@gmail.com; fernandabraz1516@gmail.com; roberta.ornellas@uesb.edu.br;
\end{abstract}

*gabrielemarisco@uesb.edu.br; http://orcid.org/0000-0002-8301-8673

\section{Resumo}

O Brasil possui ampla variedade de plantas que fornecem substâncias biológicas que podem ser utilizadas para o desenvolvimento de produtos cosméticos, contribuindo para a saúde humana e o mercado mundial. Embora existam estudos sobre essas substancias, muitos são limitados aos artigos científicos e registro de patentes. O objetivo dessa pesquisa foi realizar uma prospecção científica e tecnológica sobre o potencial de plantas com ação antimicrobiana e antioxidante para o desenvolvimento de cosméticos. Para isso foi realizado um levantamento de dados científicos utilizando o Portal de Periódicos Capes (pesquisa 1 e 2) e prospecção tecnológica (pesquisa 3), na busca de patentes na base de dados europeia Espacenet. Os resultados demonstraram 9 plantas com atividade antimicrobiana e antioxidante associada $o$ que podem torna-las ótima opção para incorporação em cosméticos. Observou-se que há produção científica por pesquisadores brasileiros sobre potencial biológico de plantas, mas poucas patentes são depositadas no Brasil ao comparar com as estrangeiras, principalmente no que se refere à descrição da ação para a produção de cosméticos.

Palavras-chave: atividade biológica, biocosméticos, recursos vegetais. 


\begin{abstract}
Brazil has a wide variety of plants that supply biological substances that can be used for the development of cosmetic products, contributing to human health and the world market. Although there are studies on these substances, many of them are retained in scientific articles and in the registration of patents. The objective of this research was to conduct a scientific and technological prospecting on the potential of plants with antimicrobial and antioxidant action for the development of cosmetics. For this, a survey of scientific data was carried out using the Capes (research 1 and 2) and technological prospection (research 3), in the search for patents in the European Espacenet database. The results showed that there is scientific production by Brazilian researchers on the biological potential of plants, but few patents are filed in Brazil when compared with foreign ones, mainly with regard to the description of the action for the production of cosmetics.
\end{abstract}

Keywords: biological activity, biocosmectics, plant resources.

\title{
1. Introdução
}

A diversidade biológica de plantas existentes no nosso planeta possui um extenso patrimônio genético, fornecendo riquezas naturais no desenvolvimento de diversos produtos e contribuindo para o mercado científico, tecnológico e econômico mundial (Pimentel et al., 2015; Bolzani., 2016).

As plantas são importantes fontes de substâncias biologicamente ativas (Funari et al., 2013), entre as quais a bioprospecção de agentes com características antioxidantes e antimicrobianas vem ganhando destaque na indústria biotecnológica, no desenvolvimento de produtos cosméticos (Miguel, 2011; Beli et al., 2020).

Pesquisas para o desenvolvimento de produtos cosméticos a partir de compostos ativos da biodiversidade vegetal tem sido uma das transformações no mercado consumidor, que busca um estilo de vida mais saudável associado a beleza, prazer, bem-estar (Miguel, 2011), e possui consciência de sustentabilidade e de preservação ao meio ambiente (Gimenez et al., 2014).

Nesse contexto, diversas plantas, principalmente espécies de regiões tropicais, podem ser utilizadas na formulação de produtos como cremes, xampus, condicionadores etc., por possuírem uma gama de princípios ativos e ter como principal característica alguma atividade biológica como antioxidantes e antimicrobianos (Miguel, 2011; Santos et al., 2018).

Os antioxidantes são substâncias eficazes na proteção do organismo contra o estresse 
oxidativo inibindo a ação de radicais livres em excesso (Santos et al., 2018). A ação antioxidante aponta eficácia no controle ou prorrogação do envelhecimento cutâneo (Jones, 2010), preservando a pele no combate a agentes endógenos e exógenos, assim, propiciando a sua incorporação a cosméticos (Henrique, 2017).

Os antimicrobianos são agentes que podem inibir o crescimento ou destruir os microrganismos (Mendonça et al., 2016), diante disso, produtos cosméticos fabricados a partir de plantas que apresentam atividade antibacteriana podem ser uma alternativa para a sua conservação, em vez de utilizar ingredientes inorgânicos que podem apresentar algum tipo de toxicidade (Amaral et al., 2010; Lombardo et al., 2020).

Nesse sentido, o desenvolvimento tecnológico de produtos cosméticos utilizando plantas é importante para valorização da diversidade biológica, ambiental, sustentável e atividade econômica. Assim, o objetivo dessa pesquisa foi realizar uma prospecção científica e tecnológica sobre o potencial de plantas com ação antibacteriana e antioxidante para o desenvolvimento de cosméticos.

\section{Material e Métodos}

Nessa pesquisa foram conduzidas três etapas metodológicas, nomeadas de pesquisa 1 , pesquisa 2 e pesquisa 3 .

A pesquisa 1 e 2 foram conduzidas a partir do Portal de Periódicos Capes, sendo as palavras-chaves pesquisas diferentes.

Na pesquisa 1, foram investigados trabalhos sobre plantas com ação antimicrobiana e antioxidante, utilizando as palavras-chave combinadas "planta, antibacteriana" e "planta, antioxidante", no idioma português. Como critérios de inclusão foram utilizados: (i) artigos publicados contra bactérias de interesse humano; (ii) artigos publicados cujos testes de ação antioxidante e antimicrobiana com plantas foram realizados; (iii) artigos publicados que não se tratavam de revisões; (iv) artigos publicados que apresentaram compatibilidade com o tema da pesquisa; e (v) trabalhos publicados nos últimos 5 anos.

$\mathrm{Na}$ pesquisa 2 foram analisados trabalhos utilizando as palavras-chave combinadas "plantas, cosméticos, atividade antibacteriana" e "plantas, cosméticos, atividade antioxidante", 
no idioma português. Os critérios de inclusão foram: (i) artigos publicados cujas plantas apresentaram atividade antioxidante e/ou antimicrobiana e potencial para produção de cosméticos; (ii) artigos publicados que não tratavam de revisões; e (iii) artigos publicados que apresentaram compatibilidade com o tema da pesquisa.

Já a pesquisa 3, de prospecção tecnológica com busca de patentes na base de dados europeia Espacenet - Europen Patent Office (https://worldwide.espacenet.com/?locale=en EP), foi realizada utilizando como palavras-chave "plantas, cosméticos e antibacteriana" e "plantas, cosméticos e antioxidante" no idioma inglês. Este banco de dado refere-se à coleção completa de pedidos de patentes em mais de 80 países, incluindo, por exemplo, os pedidos de patentes nacionais: Instituto Nacional de Propriedade Industrial (INPI) (https://www.gov.br/inpi/ptbr/servicos/patentes) e norte-americanos: United States Patent and Trademark Office (USPTO) (http://patft.uspto.gov/netahtml/PTO/search-adv.htm) e via Patent Cooperation Treaty (PCT) (https://www.wipo.int/pct/en/).

Os dados obtidos da busca foram analisados priorizando a identificação do número de trabalhos científicos e patentes encontradas, o tipo de documento, os códigos de classificação internacional, os principais depositantes e o país de origem (jurisdição). Os resultados encontrados são apresentados na forma de gráficos e tabelas para discussão das possibilidades tecnológicas apresentadas pela pesquisa.

\section{Resultados}

Na pesquisa 1 foram encontrados um total de 69 artigos sobre plantas com potencial antibacteriano, destes, apenas 17 atenderam aos critérios de inclusão. Sobre plantas com potencial antioxidante foram encontrados 30 trabalhos, dos quais 14 são compatíveis com os critérios propostos. Na pesquisa 2, ao investigar trabalhos científicos sobre plantas com ação antibacteriana e/ou antioxidante para o desenvolvimento de produtos cosméticos, o número encontrado foi menor (figura 1). 


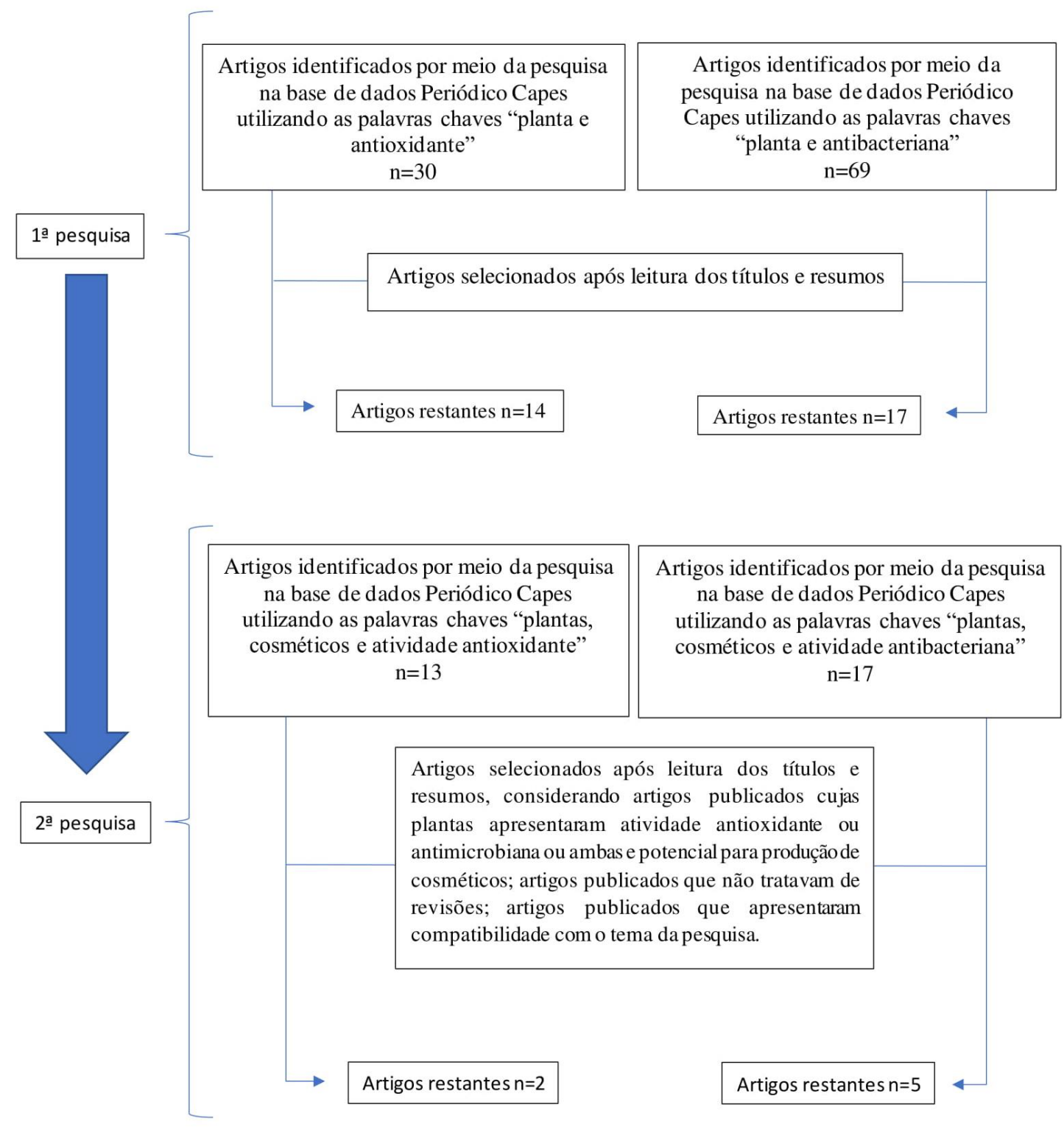

Figura 1. Artigos científicos publicados nos últimos 5 anos, sobre plantas com ação antimicrobiana e ação antioxidante, e cosméticos. Fonte: Elaborada pelos autores deste artigo (2020) 
A tabela 1 apresenta as plantas citadas nos estudos científicos das pesquisas 1 e 2 .

Tabela 1. Plantas com ação antimicrobiana e ação antioxidante, com potencial para fins cosméticos

\begin{tabular}{|c|c|c|c|}
\hline \multicolumn{2}{|c|}{ Plantas da pesquisa 1} & \multicolumn{2}{|c|}{ Plantas da Pesquisa 2} \\
\hline Atividade antibacteriana & Atividade antioxidante & Atividade antibacteriana & Atividade antioxidante \\
\hline $\mathrm{n}=20$ & $\mathrm{n}=14$ & $\mathrm{n}=10$ & $\mathrm{n}=3$ \\
\hline $\begin{array}{c}\text { Aeollanthus suaveolens } \\
\text { (Massacá) }\end{array}$ & $\begin{array}{c}\text { Byrsonima crassifólia } \\
\text { (Murici) }\end{array}$ & $\begin{array}{c}\text { Jatropha mollissima } \\
\text { (Pinhão-bravo) }\end{array}$ & $\begin{array}{c}\text { Boerhavia difusa } \\
\text { (Erva-tostão) }\end{array}$ \\
\hline Cnidoscolus quercifolius (Favela) & Chrysophyllum cainito & Cymbopogon citratus & Momordica charantia \\
\hline Copaifera reticulata (Copaíba) & (Abiu roxo) & (Capim santo) & (Melão de São \\
\hline Copaifera oblongifolia. (Copaíba- & Cnidoscolus & Euphorbia tirucalli & Caetano) \\
\hline vermelha) & chayamansa (Chaya) & (Aveloz) & Manihot esculenta \\
\hline Costus spiralis (Cana-de-macaco) & Cnidoscolus & Jatropha gossypiifolia & Crantz (Mandioca) \\
\hline Copaifera duckei (Copaíba), & quercifolius (Favela) & (Pinhão-roxo) & \\
\hline Copaifera langsdorffii (Copaíba) & Croton floribundus & Jatropha mollissima & \\
\hline Calendula officinalis (Margarida) & (Capixingui) & Myrciaria cauliflora & \\
\hline Magnolia schiedeana (Magnólia- & Hylocereus polyrhizus & (Jabuticaba) & \\
\hline Branca) & (Pitaya vermelha) & Citrus sinensis (Laranja) & \\
\hline Malpighia emarginata (Acerola) & Luehea paniculata & Ocimum basilicum & \\
\hline Mangifera indica (manga) & (Açoita cavalo) & (Manjericão) & \\
\hline Mentha piperita (Hortelã-Pimenta) & Morus alba (Amora & Psidium guajava & \\
\hline Myrcia tomentosa (Goiabinha) & branca) & (Goiabeira) & \\
\hline Passiflora maliformis (Maracujá- & Peltogyne mexicana & Syzygium cumini & \\
\hline Amarelo) & (Morao) & (Jabelão) & \\
\hline Persea americana (Abacate) & Plantago coronopus & & \\
\hline Psittacanthus linearis & (Diabelha) & & \\
\hline Rhaphiodon echinus (Beton) & Psidium cattleianum & & \\
\hline Roscoe Costaceae (Cana-do-brejo) & (Araçá-rosa) & & \\
\hline Citrus sinensis (Laranja) & Schinopsis brasiliensis & & \\
\hline \multirow[t]{5}{*}{ Senna occidentalis (Fedegoso) } & (Braúna-do-sertão) & & \\
\hline & Solanum sessiliflorum & & \\
\hline & (Cubiu) & & \\
\hline & Ugni molinae & & \\
\hline & (Murtilla) & & \\
\hline
\end{tabular}

Referente a pesquisa 3, foram encontradas 11 patentes relacionadas com plantas com potencial antibacteriano e 17 plantas com potencial antioxidante para o desenvolvimento de produtos cosméticos (tabela 2). 
Tabela 2. Patentes encontradas sobre plantas com potencial antimicrobiano e antioxidante para fins cosméticos.

\begin{tabular}{|c|c|c|c|}
\hline Códigos & Espécies vegetais estudadas & Produto/aplicação & Origem \\
\hline KR970007366B1 & $\begin{array}{c}\text { Sasa borealis e Sesamum indicum } \\
\text { (gergelim) }\end{array}$ & $\begin{array}{l}\text { A formulação é útil como } \\
\text { aditivo para cosméticos. }\end{array}$ & Coréia \\
\hline CA2021637A1 & Krameria sp. ou Eupomatia sp. & $\begin{array}{l}\text { Os extratos podem ser usados } \\
\text { na formulação de cosméticos. }\end{array}$ & Milão \\
\hline WO02062362A2 & $\begin{array}{c}\text { Annona squamosa Lnn., Eugenia } \\
\text { macrophylla Lam., Euphoria longana } \\
\text { Lam. E Sandoricum koetjapa }\end{array}$ & $\begin{array}{l}\text { Os extratos podem ser usados } \\
\text { na formulação de cosméticos. }\end{array}$ & Japão \\
\hline EP0464297A1 & $\begin{array}{c}\text { Perilla frutescens, Lavandula vera, } \\
\text { Isodon japonicus, Rosmarinus } \\
\text { officinalia, Melissa officinalis, Lamium } \\
\text { album, Salivia miltiorhiza e Coleus } \\
\text { forskohlii }\end{array}$ & $\begin{array}{l}\text { Cosmético antibacteriano com } \\
\text { baixa irritação ao cabelo e a } \\
\text { pele. }\end{array}$ & Japão \\
\hline KR20188811618A & Ginseng vermelho & $\begin{array}{l}\text { Pode ser usado na produção de } \\
\text { cosméticos. }\end{array}$ & Coréia \\
\hline JP2006055038A & Psidium guajava (goiaba) & $\begin{array}{l}\text { Pode ser usado na formulação } \\
\text { de cosmético. }\end{array}$ & Japão \\
\hline CN103462860A & $\begin{array}{c}\text { Murraya paniculata, Stemona } \\
\text { tuberosa, Eleutherine plicata e Litsea } \\
\text { cubeba }\end{array}$ & $\begin{array}{l}\text { Aplicado do campo dos } \\
\text { cosméticos. }\end{array}$ & $\begin{array}{l}\text { China do } \\
\text { Sul }\end{array}$ \\
\hline JP2009001539A & $\begin{array}{l}\text { Lavandula (lavanda), Calendula } \\
\text { officinalis (Margarida), Radix } \\
\text { scutellariae, Salvia rosmarinus } \\
\text { (alecrim), Syzygium aromaticum, } \\
\text { Glycyrrhiza glabra (alcaçuz) }\end{array}$ & $\begin{array}{l}\text { Conservante composto para } \\
\text { cosmético. }\end{array}$ & China \\
\hline CN106420466A & $\begin{array}{l}\text { Radix sophorae flavescentis, Radix et } \\
\text { Rhizoma rhei, Rhizoma belamcandae, } \\
\text { Radix scutellariae e Rhizoma polygoni }\end{array}$ & $\begin{array}{l}\text { Pode ser adicionado em } \\
\text { shampoo (anticaspas), espuma } \\
\text { de banho, sabonete líquido, } \\
\text { sabão antibacteriano e } \\
\text { cosméticos. }\end{array}$ & China \\
\hline CN103858945A & Apocynum venetum & Aditivos cosméticos. & China \\
\hline CN106389171A & $\begin{array}{c}\text { Matricaria chamomilla (camomila), } \\
\text { Lavandula (lavanda), Rosacear (rosa), } \\
\text { Aloe vera. }\end{array}$ & Produto para a pele. & China \\
\hline
\end{tabular}


...Continuação da Tabela 2

\section{PLANTAS, COSMÉTICOS e ANTIOXIDANTE}

\begin{tabular}{|c|c|c|c|}
\hline CN108158884A & $\begin{array}{l}\text { Extrato de Brasenia schreberi e o } \\
\text { extrato de Caulerpa lentillifera }\end{array}$ & $\begin{array}{l}\text { Composição cosmética de } \\
\text { matéria-prima com eficácia } \\
\text { inibidora de alergias e } \\
\text { retardadora do } \\
\text { envelhecimento. }\end{array}$ & China \\
\hline
\end{tabular}

\begin{tabular}{|c|c|c|c|}
\hline CN107625677A & $\begin{array}{l}\text { Chá, sementes e extrato de plantas } \\
\text { mistas }\end{array}$ & $\begin{array}{l}\text { Fórmula extraída de plantas } \\
\text { mistas, para incorporação em } \\
\text { alimentos, bebidas, agentes } \\
\text { aromatizantes, cosméticos e } \\
\text { medicamentos. }\end{array}$ & China \\
\hline CN106727061A & $\begin{array}{l}\text { Extrato de amoreira, extrato de } \\
\text { wolfberry e extrato de erva Humifusa } \\
\text { euphorbia }\end{array}$ & $\begin{array}{l}\text { Essência para } \\
\text { rejuvenescimento da pele, com } \\
\text { ação antioxidante. }\end{array}$ & China \\
\hline CN106473987 & $\begin{array}{l}\text { Extrato de Alnifolia, amelanchier nutt, } \\
\text { extrato de Centella asiatica e extrato } \\
\text { de Flos sophorae immaturus. }\end{array}$ & $\begin{array}{l}\text { Composição de clareamento } \\
\text { da pele e antienvelhecimento } \\
\text { com ação antioxidante. }\end{array}$ & China \\
\hline CN105326902A & Extrato de Michelia yunnanensis & $\begin{array}{l}\text { Pode ser aplicada em } \\
\text { cosméticos para a pele } \\
\text { antirrugas, clareadores e } \\
\text { bloqueadores solares. }\end{array}$ & China \\
\hline KR20120108930A & Suco ou extrato da maçã & $\begin{array}{l}\text { Material cosmético contendo } \\
\text { suco ou extrato de maçã. Ação } \\
\text { antioxidante. }\end{array}$ & Japão \\
\hline JP2011051992A & $\begin{array}{l}\text { Cascas de árvores de Acacia mearnsii, } \\
\text { A. mangium, A. dealbata, A. decurrens e } \\
\text { A. pycnantha e extratos solventes das } \\
\text { cascas das árvores }\end{array}$ & $\begin{array}{l}\text { As cascas de árvores das plantas } \\
\text { do gênero Acácia e seus extratos } \\
\text { solventes têm uma alta ação de } \\
\text { radicais livres, portanto, podem } \\
\text { ser utilizadas em alimentos, } \\
\text { medicamentos, cosméticos etc. }\end{array}$ & Japão \\
\hline CN101906132A & Sementes de aipo & $\begin{array}{l}\text { Os polipeptídios preparados } \\
\text { com o método podem ser } \\
\text { utilizados como aditivos na } \\
\text { preparação de alimentos e } \\
\text { cosméticos. Possuem ação } \\
\text { antioxidante. }\end{array}$ & China \\
\hline
\end{tabular}


...Continuação da Tabela 2

\begin{tabular}{|c|c|c|c|}
\hline KR20020070553A & Phyllostachys reticulata & $\begin{array}{l}\text { O composto (1,2-di-orto- } \\
\text { ferroilglicerol com atividade } \\
\text { antioxidante) é utilizado para } \\
\text { cosméticos excelentes nos } \\
\text { efeitos de clareamento da pele e } \\
\text { um inibidor da melanogênese ou } \\
\text { um agente da pele de uso } \\
\text { externo excelente na } \\
\text { estabilidade da pele. }\end{array}$ & $\begin{array}{l}\text { República } \\
\text { da Coréia }\end{array}$ \\
\hline KR20010088120A & $\begin{array}{l}\text { extrato de misturas vegetais Scutellaria } \\
\text { baikalensis, Paeonia suffruticosa e } \\
\text { Styphnolobium japonicum }\end{array}$ & $\begin{array}{l}\text { Composição cosmética de } \\
\text { clareamento de pele que possui } \\
\text { excelente ação antioxidante. }\end{array}$ & $\begin{array}{l}\text { República } \\
\text { da Coréia }\end{array}$ \\
\hline JP2008013461A & $\begin{array}{l}\text { Extrato de Gallae rhois, Zanthoxyli } \\
\text { bungeanum, Flos sophorae, laurier, } \\
\text { manjericão e tomilho }\end{array}$ & $\begin{array}{l}\text { Fornecimento de um composto } \\
\text { antioxidante que estabilize a } \\
\text { oxidação podendo ser aderido a } \\
\text { cosméticos de cuidado com a } \\
\text { pele para prolongar o uso. }\end{array}$ & Japão \\
\hline JP2002322191A & $\begin{array}{l}\text { Extrato das plantas da família do } \\
\text { gergelim }\end{array}$ & $\begin{array}{l}\text { Novo glicosídeo de sesaminol e } \\
\text { um antioxidante natural solúvel } \\
\text { em água que contenha o } \\
\text { glicosídeo de sesaminol com } \\
\text { aplicação em cosméticos. }\end{array}$ & Japão \\
\hline JPH11199427A & Extrato das folhas de Citrus & $\begin{array}{l}\text { Composição antioxidante que } \\
\text { possa ser utilizada em } \\
\text { cosméticos. }\end{array}$ & Japão \\
\hline JPH10182336A & $\begin{array}{c}\text { Psoralea pubescens e P. mexicana (flor, } \\
\text { semente, folha, raiz) }\end{array}$ & $\begin{array}{l}\text { Agente antioxidante com } \\
\text { aplicação em diversos } \\
\text { cosméticos. }\end{array}$ & Japão \\
\hline JPH1150050A & $\begin{array}{l}\text { Pogostemon viverita, Pogostemon } \\
\text { vivertia, Bidens pilosa, Eupatorium } \\
\text { triplinerve, Mikania humilifollia, Lippia } \\
\text { alba, Aspidosperma excelsum, Borreria } \\
\text { verticillata, Cissus sicyoides, Leucas } \\
\text { martinicensis e Phtirusa pyrifolia }\end{array}$ & $\begin{array}{l}\text { Antioxidante estável com } \\
\text { aplicação em cosméticos. }\end{array}$ & Japão \\
\hline EP0464297A1 & Krameria sp. e Eupomatia sp. & $\begin{array}{l}\text { Complexos de extratos dessas } \\
\text { plantas que podem apresentar } \\
\text { atividade antioxidante e } \\
\text { aplicável em cosméticos. }\end{array}$ & $\begin{array}{c}\text { Organização } \\
\text { Europeia de } \\
\text { Patentes } \\
\text { (OPE/EPO) }\end{array}$ \\
\hline DE10113205A1 & Resíduos de plantas e vegetais diversos & Antioxidantes. & Alemanha \\
\hline
\end{tabular}

Fonte: Elaborada pelos autores deste artigo (2020) 
De acordo com as pesquisas 1 e 2 , observou-se que $78,95 \%$ dos trabalhos foram realizados em universidades brasileiras. E apesar de constatar que há estudos científicos desenvolvidos sobre plantas com atividade antioxidante e antibacteriana no Brasil, verifica-se que a maioria das patentes desta área são de origem chinesa e nenhuma do Brasil foi encontrado (figura 2).

Figura 2. Países referentes a patentes de plantas com (A) atividade antioxidante e potencial cosmético; (B) plantas com atividade antibacteriana com potencial cosmético.

A

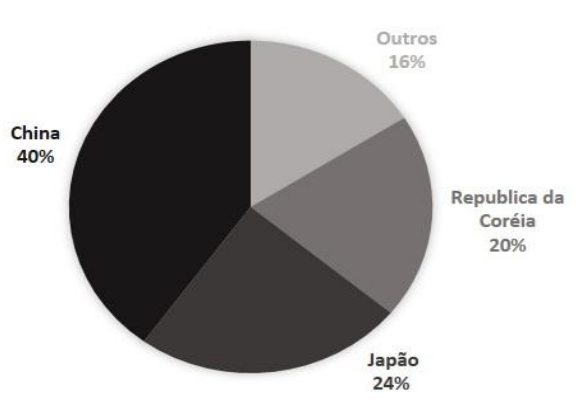

B

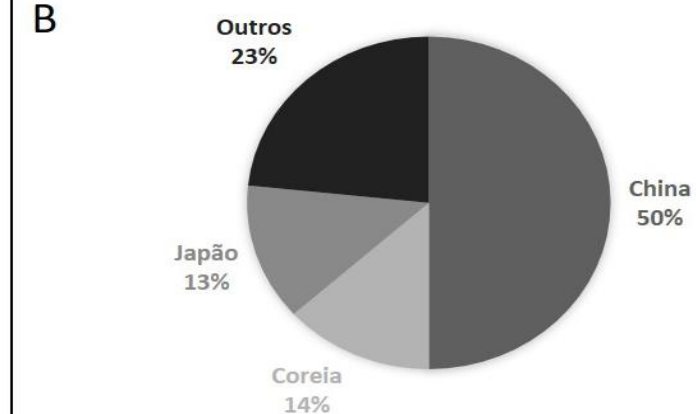

Fonte: Elaborada pelos autores deste artigo (2020)

A partir das análises feitas nessa pesquisa, verificou-se que 9 plantas possuem ação antimicrobiana e antioxidante associadas (Tabela 3).

Tabela 3. Plantas com ação associada como antioxidante e antimicrobiana.

Plantas com ação antioxidante e atividade

\section{Referência}

antimicrobiana

\begin{tabular}{lc}
\hline Croton floribundus (Capixingui) & Barth et al. (2018) \\
Ugni molinae (Murtilla) & Junqueira-Gonçalves et al. (2015) \\
Schinopsis brasiliensis (Braúna-do-sertão) & Lima-Saraiva et al. (2017) \\
Cnidoscolus quercifolius (Favela) & Paredes et al. (2016) \\
Psidium cattleianum (Araçá) & Scur et al. (2016) \\
Persea americana (Abacate) & Amado et al. (2019) \\
Malpighia emarginata (Acerola) & Marques et al. (2017) \\
Aeollanthus suaveolens (Massacá) & Da Silva Ramos et al. (2017) \\
Mentha piperita (Hortelã-Pimenta) & Martins et al. (2016) \\
\hline
\end{tabular}

Fonte: Elaborada pelos autores deste artigo (2020) 


\section{Discussão}

Embora haja produção científica por pesquisadores brasileiros, verifica-se que não são priorizadas as patentes. Os depósitos de patentes no Brasil estão diretamente relacionados com os investimentos em ciência e tecnologia. Nos anos de 2015 a 2016 observou-se regressão nos gastos com áreas que estão fortemente correlacionadas: Atividades Científicas e Técnicas Correlatas (ACTC) e Pesquisa e Desenvolvimento (P\&D), permitindo a conclusão de que a redução dos investimentos na pesquisa pode afetar diretamente o depósito de patentes, contribuindo para sua redução gradativa (da Silva Vaz, 2019).

Associado a isso, para Bolzani (2016) um dos fatores que retarda as inovações no que se refere à biodiversidade brasileira, como fonte para a produção de formulações naturais estão relacionadas com a burocracia ao acesso ao patrimônio genético, aos saberes tradicionais, proteção e à repartição de benefícios, dificultando estudos na área da diversidade biológica.

Embora o uso e produção de plantas possui apelo ambiental, sustentável e econômico, ainda são pouco explorados no Brasil, fato que pode estar associado a aspectos envolvendo certificação, o preço elevado e a falta de interesse em questões concernentes a sustentabilidade aplicada ao desenvolvimento de cosméticos (Yamada, 2013).

Domingos et al. (2016) afirmam que as produções com matérias-primas vegetais demandam procedimentos mais modernos e tecnológicos, o Brasil, porém, ainda é iniciante nessa temática, com poucas buscas e investimentos no uso de ativos vegetais nativos, tornando mais comum a exportação desse material para países que disponham dos meios de produção. Porém, as indústrias cosméticas estão adotando os recursos de origem vegetal de modo a obter resultados positivos para elas próprias, consumidores e meio ambiente.

Entretanto, Coimbra (2017) afirma que a formulação natural em cosméticos já é uma realidade no Brasil e grande parte dos brasileiros tem considerado o impacto ambiental dos produtos que consomem. Há uma maior preocupação e sensibilização aos reais danos à saúde e ao meio ambiente ocasionados por ingredientes químicos (Bispo, 2008). Produtos que utilizam de materiais naturais além de serem biodegradáveis com mínimos impactos ao meio ambiente,-possuem menor incidência a ocorrência de alergias e efeitos colaterais (Lopes, 2010). 
Amado et al. (2019) afirma que as atividades antioxidante e antibacteriana dos produtos naturais podem mudar de acordo a diversidade vegetal, bem como a parte da planta que é utilizada, então surge a necessidade de analisar partes diferentes de uma mesma planta e ainda conferir suas propriedades biológicas. Também existem poucos estudos comparando ações conjuntas para a atividade antimicrobiana e antioxidante de plantas com potencial cosmético.

O desenvolvimento de cosméticos a partir de plantas com ações antimicrobiana e antioxidante pode atender à demanda do mercado, oferecendo uma alternativa interessante no desenvolvimento de um produto. A atividade antioxidante contribui com a diminuição dos radicais livre, reduzindo o envelhecimento precoce (Santos et al., 2018), enquanto a atividade antimicrobiana contribui para a conservação do produto evitando o crescimento e proliferação de microrganismos. Esses aspectos contribuem com menor impacto ambiental diminuindo a concentração de aditivos sintéticos utilizados pela indústria, reduzindo riscos à saúde humana (Lopes et al., 2010; Silvestri et al., 2010).

Conclui-se que, ainda não existem números expressivos de trabalhos acadêmicos publicados envolvendo plantas e seu uso nos cosméticos, mas há um número bastante significativo de documentos de patentes, principalmente referentes à descrição de eventual ação para a produção de cosméticos. Porém, em sua maioria, as patentes são internacionais, demonstrando que há pouco direcionamento das pesquisas no Brasil para patentes e desenvolvimento de produtos naturais nas indústrias de cosméticos nacionais.

Salienta-se a urgência da continuidade das pesquisas básicas até o desenvolvimento final de produtos no Brasil, para valorização científica, bem como garantir o direito dos inventores de beneficiar-se comercialmente da sua produção intelectual, incentivando a continuidade de pesquisas da biodiversidade e os recursos vegetais. Os produtos cosméticos apresentam potencial tecnológico e econômico, tendo em vista que cada vez mais consumidores têm interesse em usar produtos de origem natural.

\section{Referencias}

Amado, D. A. V.; Helmann, G. A. B.; Detoni, A. M. et al. (2019). Antioxidant and antibacterial activity and preliminary toxicity analysis of four varieties of avocado (Persea americana Mill.). Brazilian Journal of Food Technology, v. 22, e2018044, 11p. 


\section{https://doi.org/10.1590/1981-6723.04418}

Amaral, L. F. B. Avaliação da eficácia antimicrobiana do monoéster de C-8 xilitol como alternativa conservante para produtos cosméticos. Dissertação-mestrado, Universidade Estadual de Campinas, Faculdade de Ciências Médicas, Campinas, SP. 108p. 2010. Disponível em: http://repositorio.unicamp.br/jspui/handle/REPOSIP/311649. Acessado em 1 de março de 2021 .

Barth, E. F.; Pinto, L. S.; Dileli, P. et al. (2018). Biological screening of extracts from leaf and stem bark of Croton floribundus Spreng. (Euphorbiaceae). Brazilian Journal of Biology, v. 78, n. 4, p. 601-608. https://doi.org/10.1590/1519-6984.166522

Beli, C. M.; Mageste, J. M.; Taketani, N. F. (2020). Bioprospecção de enzimas para cosmética: seu impacto na biotecnologia. Revista Ensaios Pioneiros, v. 3, n. 2, p. 10-24. https://doi.org/10.24933/rep.v3i2.199

Bispo, M. (2008). Cosméticos verdadeiramente orgânicos. Cosmetics \& Toiletries Brasil, p. 50-52.

Bolzani, V. D. S. (2016). Biodiversidade, bioprospecção e inovação no Brasil. Ciência e Cultura, v. 68, n. 1, p. 04-05. http://dx.doi.org/10.21800/2317-66602016000100002

Coimbra, A.R. (2017). Preservação de cosméticos naturais. Cosmetics \& Toiletries Brasil, v. 29 , n. 2, p. 52-55.

da Silva Ramos, R.; Rodrigues, A. B. L.; Farias, A. L. F. et al. (2017). Chemical composition and in vitro antioxidant, cytotoxic, antimicrobial, and larvicidal activities of the essential oil of Mentha piperita L. (Lamiaceae). The Scientific World Journal, v. 2017. https://doi.org/10.1155/2017/4927214

Da Silva Vaz, V. H. (2019). Avaliação do depósito de patentes no brasil a partir dos dispêndios públicos em ciência e tecnologia. PIDCC, v. 13, n. 3, p. 84-92.

Domingos, B.G.; Moraes, C.A.P; Nicoletti, M.A; ITO, R.K. (2016). A biodiversidade na indústria de cosméticos brasileira. Cosmetics \& Toiletries Brasil, v. 28, n. 5, p. 68-74.

Funari, C. S.; Castro-Gamboa, I.; Cavalheiro, A. J. et al. (2013). Metabolômica, uma abordagem otimizada para exploração da biodiversidade brasileira: estado da arte, perspectivas e desafios. Química Nova, v. 36, n. 10, p. 1605-1609. https://doi.org/10.1590/S0100$\underline{40422013001000019}$

Gimenez, F.; Valim Dias, L. D. C.; Higuchi, C. T. (2014). Estudo da consciência do consumidor com relação aos ativos sintéticos e ativos naturais presentes nos cosméticos. InterfacEHSRevista de Saúde, Meio Ambiente e Sustentabilidade, v. 8, n. 3.

Henrique, A. D. S. \& Lopes, G. C. (2017). Biodiversidade e a indústria de cosméticos: O uso dos flavonóides contra o envelhecimento cutâneo. Revista Uningá Review, v. 29, n. 2, p. 58-63.

Jones, G. (2010). Beauty imagined: a history of the global beauty industry. Oxford University Press on Demand. $412 p$

Junqueira-Gonçalves, M. P.; Yáñez, L.; Morales, C. et al. (2015). Isolation and characterization 
of phenolic compounds and anthocyanins from murta (Ugni molinae Turcz.) fruits. Assessment of antioxidant and antibacterial activity. Molecules, v. 20, n. 4, p. 5698-5713. https://doi.org/10.3390/molecules20045698

Lima-Saraiva, S. R. G. D.; Oliveira, F. G. D. S.; Junior, R. G. D. O. et al. (2017). Chemical analysis and evaluation of antioxidant, antimicrobial, and photoprotective activities of Schinopsis brasiliensis Engl. (Anacardiaceae). The Scientific World Journal, v. 2017. https://doi.org/10.1155/2017/1713921

Lombardo, M. (2020). Estabilização e conservação de formulações farmacêuticas e cosméticas: aspectos de qualidade e de segurança. Journal of Applied Pharmaceutical Sciences, n. 7, p. 4355.

Lopes, R. H. \& Cavalcante, K. (2010). A Amazônia como apelo de mercado e estoque de matéria-prima para a indústria de biocosméticos: ficção ou realidade. Encontro Nacional de Engenharia de Produção, 30.

Marques, T. R.; Caetano, A. A.; Rodrigues, L. M. A. et al. (2017). < b > Characterization of phenolic compounds, antioxidant and antibacterial potential the extract of acerola bagasse $\begin{array}{llllll}\text { flour. Acta Scientiarum. } & \text { Technology, v. } 39, \quad \text { n. } & 2, & \text { p. 143-148. }\end{array}$ https://doi.org/10.4025/actascitechnol.v39i2.28410

Martins, R. L.; Simões, R. C.; Rabelo, É. D. M. et al. (2016). Chemical composition, an antioxidant, cytotoxic and microbiological activity of the essential oil from the leaves of Aeollanthus suaveolens Mart. ex Spreng. PloS one, v. 11, n. 1, e0166684. https://doi.org/10.1371/journal.pone.0166684

Mendonça, A. T.; Carvalho, A. R.; Ferreira, M. C. et al. (2016). A utilização dos extratos hidroalcoólico e alcoólico de Eugenia uniflora L. como agente antibacteriano. Revista da Universidade Vale do Rio Verde, v. 14, n. 1, p. 826-833. http://dx.doi.org/10.5892/ruvrd.v14i1.3019

Miguel, L. M. (2011). Tendências do uso de produtos naturais nas indústrias de cosméticos da França. Revista Geográfica de América Central, v. 2, pp. 1-15.

Paredes, P. F. M.; Vasconcelos, F. R.; Paim, R. T. T. et al. (2016). Screening of bioactivities and toxicity of Cnidoscolus quercifolius Pohl. Evidence-Based Complementary and Alternative Medicine, 2016. https://doi.org/10.1155/2016/7930563

Pimentel, V. P.; Vieira, V. A. M.; Mitidieri, T. L. et al. (2015). Biodiversidade brasileira como fonte da inovação farmacêutica: uma nova esperança?

Santos, J. A. S.; Sena, T. J. O.; da Costa, M. L. A. et al. (2018). Estudo do potencial antioxidante da Anacardium occidentales L. e determinação de seus compostos fenólicos. Diversitas Journal, v. 3, n. 2, p. 455-474. https://doi.org/10.17648/diversitas-journal-v3i2.637

Scur, M. C.; Pinto, F. G. S.; Pandini, J. A. et al. (2016). Antimicrobial and antioxidant activity of essential oil and different plant extracts of Psidium cattleianum Sabine. Brazilian Journal of Biology, v. 76, n. 1, p. 101-108. https://doi.org/10.1590/1519-6984.13714

Silvestri, J. D. F. (2010). Perfil da composição química e atividades antibacteriana e 
antioxidante do óleo essencial do cravo-da-índia (Eugenia caryophyllata Thunb.). Revista Ceres, v. 57, n. 5, p. 589-594. https://doi.org/10.1590/S0034-737X2010000500004

Yamada, D. A. S.; de Lima, L. R.; do Nascimento Santos, S. et al. (2013) Discussão crítica da legislação orgânica aplicada aos produtos cosméticos sustentáveis e investigação científica na prática do consumo. InterfacEHS., v. 8, n. 3, p. 3-18.

\section{Direitos Autorias (copyrights)}

\section{Financiamento}

Este estudo foi financiado por bolsas de iniciação científica do CNPq e UESB.

\section{Conflitos de interesse}

Todos os autores declaram não haver conflito de interesses.

\section{Aprovação do comitê de ética}

Não aplicável.

\section{Disponibilidade dos dados de pesquisa}

Todos os dados gerados ou analisados neste estudo estão incluídos no manuscrito.

\section{Contribuição dos autores}

Idealização: Marisco, G; Investigação/execução da pesquisa: Costa, APL, Jesus, FB; Análise formal: Marisco, G, Ornellas, R; Metodologia: Costa, APL, Jesus, FB; Redação - revisão e edição: Marisco, G, Ornellas, R.

Os autores concedem direitos autorais sobre manuscrito aprovado com exclusividade de publicação para Revista Textura em formato eletrônico, incluindo imagens e conteúdo para divulgação do artigo, inclusive nas redes sociais da Revista Textura. 